\title{
EXISTEM RELAÇÕES ENTRE QUALIDADE DE VIDA NO TRABALHO, COMPROMETIMENTO ORGANIZACIONAL E ENTRINCHEIRAMENTO ORGANIZACIONAL?
}

\author{
ARE THERE RELATIONSHIPS AMONG QUALITY OF WORK \\ LIFE, ORGANIZATIONAL COMMITMENT AND \\ ORGANIZATIONAL ENTRENCHMENT?
}

\author{
¿EXISTEN RELACIONES ENTRE CALIDAD DE VIDA EN EL \\ TRABAJO, COMPROMISO ORGANIZACIONAL Y \\ ATRINCHERAMIENTO ORGANIZACIONAL?
}

Artigo aprovado em fast-track do XXI SEMEAD

Jaqueline Cavalcante Milhome

Mestre em Administração - NPGA/UFBA,

Salvador, Brasil

Doutoranda em Administração - NPGA/UFBA,

Salvador, Brasil

jaquemilhome@yahoo.com.br

Diva Ester Okazaki Rowe

Doutorado em Administração - NPGA/UFBA, Salvador, Brasil

Professora associada - NPGA/UFBA, Salvador,

Brasil

diva@ufba.br

\section{Marcos Gilberto Dos-Santos}

Mestre em Administração - UNIFACS, Salvador,

Brasil

Professor EBTT - Instituto Federal de Educação,

Ciência e Tecnologia da Bahia (IFBA), Paulo

Afonso, Brasil

marcosgilberto74@gmail.com
Contextus

ISSN 1678-2089

ISSNe 2178-9258

Organização: Comitê Científico Interinstitucional Editor Científico: Diego de Queiroz Machado Editor Executivo: Carlos Daniel Andrade Avaliação: double blind review pelo SEER/OJS

Recebido em 29/10/2018 Aceito em 29/10/2018 $2^{a}$ versão aceita em 04/11/2018

\section{RESUMO}

Uma vez reconhecida a participação substancial do trabalhador no resultado organizacional, evidencia-se que a percepção dele sobre diversos aspectos organizacionais pode influenciar e ser influenciada pelo tipo de vínculo que ele desenvolve com a organização. Nesse contexto, o presente estudo visa analisar as possíveis relações que podem se estabelecer entre a qualidade de vida no trabalho e os vínculos comprometimento e entrincheiramento organizacional. Para tanto, foi realizada uma pesquisa quantitativa e explicativa, cujo questionário foi composto por três escalas validadas, cada uma relacionada a um dos construtos investigados. A partir de uma amostra de 187 trabalhadores, os dados foram analisados com regressões múltiplas. Os resultados confirmaram algumas hipóteses e contribuíram para refletir sobre a importância do vínculo que o trabalhador desenvolve com a organização e, especificamente, o modo como os tipos de vínculo podem contribuir para a percepção acerca das práticas e posicionamentos organizacionais.

Palavras-chave: comprometimento; entrincheiramento; qualidade de vida no trabalho; vínculos com a organização; comportamento organizacional. 


\begin{abstract}
Employees influence substantially the organizational outcomes. Their point of view about some organizational elements may influence, and also be influenced by, their kind of links developed to the organization. Within that context, this research aims to analyze the possible relationships between quality of work life, organizational commitment and organizational entrenchment. With that in view, an explanatory, quantitative research was carried out that used a survey composed of three validated scales, each related to one of the examined constructs. The sample was 187 employees and the data were analyzed by means of multiple regression.. The results confirmed some hypotheses and contributed to reflex about both how important the link developed by the worker to the organization is and how the kinds of link can play a role with respect to employees' perception about organizational practices and positions.
\end{abstract}

Keywords: commitment; entrenchment; quality of work life; links towards organizations; organizational behavior.

\title{
RESUMEN
}

Una vez reconociendo la participación sustancial del trabajador en el resultado organizacional, se evidencia que la percepción acerca de diversos aspectos organizacionales puede influenciar y ser influenciada por el tipo de vínculo que el trabajador desarrolla con la organización. En ese contexto, el presente estudio tiene como objetivo analizar las posibles relaciones que pueden establecerse entre la calidad de vida en el trabajo y los vínculos compromiso y atrincheramiento organizacionales. Para esto, fue realizada una investigación cuantitativa y explicativa, cuyo cuestionario fue compuesto por tres escalas validadas, cada una relacionada a uno de los constructos investigados. A partir de una muestra de 187 trabajadores, los datos fueron analizados con regresiones múltiples. Los resultados confirmaron algunas hipótesis probadas y contribuyen a reflexionar sobre la importancia del vínculo que el trabajador desarrolla con la organización y, específicamente, cómo esos vínculos pueden contribuir para la percepción acerca de las prácticas y posicionamientos organizacionales.

Palabras clave: compromiso; atrincheramiento; calidad de vida en el trabajo; vínculos con la organización; comportamiento organizacional.

\section{INTRODUÇÃO}

A psicologia organizacional tem ampliado seus estudos ao analisar interações múltiplas que melhorem a qualidade de vida e o bem-estar do trabalhador, sem comprometer a produtividade e os lucros das organizações. Nesse sentido, tem abordado atitudes e comportamentos do profissional em seu ambiente, bem como a relação daquele com a organização (ZANELLI; BASTOS; RODRIGUES, 2014).

Vêm sendo amplamente estudadas as relações afetiva (comprometimento organizacional) e instrumental (entrincheiramento organizacional) como contrapontos mútuos ou elementos complementares, bem como o que as explica e o que delas se origina (BALSAN et al., 2015; KIM; BEEHR, 2018; MEYER; HERSCOVITCH, 2001; MEYER; STANLEY; PARFYONOVA, 2012). No entanto, a percepção das práticas organizacionais ligadas a essas diferentes espécies de vínculo é uma lacuna a ser estudada. A presente pesquisa aceita essa tarefa e, para tanto, assume que o modo de perceber a qualidade de vida no trabalho pode influenciar a natureza do vínculo que o trabalhador desenvolve com a organização. Diferentes níveis de vínculos diversos podem acarretar comportamentos e atitudes dos trabalhadores alinhados a resultados mais ou menos interessantes para a organização. 
Partindo dessa premissa, esta pesquisa tem por objetivo analisar a relação entre, de um lado, o comprometimento e o entrincheiramento organizacionais e, do outro, a qualidade de vida no trabalho, enquanto antecedentes e enquanto consequentes. Dessa forma, será possível compreender o quanto o comprometimento e o entrincheiramento organizacionais influenciam e são influenciados pelo modo como os trabalhadores percebem a sua qualidade de vida na empresa. Para tanto, pergunta-se: em que medida os vínculos do indivíduo - comprometimento e entrincheiramento organizacionais - influenciam e são influenciados pela percepção do profissional de sua qualidade de vida no trabalho?

A qualidade de vida no trabalho diz respeito a um conjunto de elementos oferecidos ao trabalhador, de forma a atuar nos seus aspectos físico, psicológico, social, de saúde e de recompensas. Conjuntamente, esses elementos contribuem para o bem-estar do indivíduo, a partir das experiências no seu ambiente profissional que vão impactar, sob a ótica tanto dele próprio quanto do mercado: sua satisfação, seu desempenho e sua imagem da organização.

Experiências e emoções positivas lá vividas revelam-se importantes para a natureza do vínculo que o trabalhador desenvolve. Experiências positivas em geral (KIM; BEEHR, 2018) e aquelas em torno da liderança (ALZUBI, 2018; DEMIRTAS; AKDOGAN, 2015; KARAKUŞ, 2018) podem promover o envolvimento com a firma, inibir a intenção de saída e influenciar o nível de comprometimento, fortalecendo comportamentos desejados pela organização e inibindo os indesejados.

Quanto ao entrincheiramento, identifica-se uma lacuna referente à influência sobre ele exercida por aquelas experiências provenientes da organização. Tal como corroboram Meireles (2015), Rodrigues (2009) e Rodrigues e Bastos (2015), a atuação do trabalhador é influenciada negativamente pela sensação de aprisionamento oriunda do vínculo instrumental. $O$ que a organização oferece ao trabalhador impacta a forma como ele se vincula a ela. Diante disso, a presente pesquisa buscou investigar a influência das práticas oferecidas pela organização, em favor da qualidade de vida no trabalho, sobre ambos os vínculos organizacionais: comprometimento e entrincheiramento.

O nível de qualidade de vida variará a partir da percepção dos diversos elementos organizacionais. Tal percepção, por sua vez, vai se transformar em função de um sentimento de afeto ou de aprisionamento pela organização, que direciona a natureza de vínculo estabelecido com ela. Essa relação é desvelada na presente pesquisa analisando-se a influência dos vínculos sobre a qualidade de vida no trabalho, considerando todas as dimensões que a compõem. 


\section{VÍNCULOS ORGANIZACIONAIS E QUALIDADE DE VIDA NO TRABALHO}

Trabalhadores se vinculam às organizações por diversos motivos. O afeto, o desejo de permanecer e o orgulho de integrar a organização caracterizam o comprometimento organizacional. Por outro lado, uma ligação por necessidade, devido a possíveis perdas decorrentes da saída da organização, denomina-se entrincheiramento organizacional.

Trata-se de vínculos diferentes, porém não opostos. Algumas pesquisas foram desenvolvidas a fim de analisar o nível de correlação entre o comprometimento organizacional, segundo a perspectiva afetiva, e o entrincheiramento organizacional. Estes construtos, de acordo com análises de Pearson (MEIRELES, 2015), de Spearman (BALSAN et al., 2015) e de Kendall (MILHOME; ROWE, 2017), apresentam correlação baixa entre si, porém estatisticamente válida. Balsan et al. (2015) e Rodrigues (2009) evidenciam que estes vínculos existem concomitantemente no trabalhador, em diferentes níveis.

A natureza e a intensidade tais vínculos podem variar de acordo com diversos aspectos, sejam eles pessoais - do trabalhador - ou organizacionais (BALSAN et al., 2015; MEIRELES, 2015; KARAKUŞ, 2018; KIM; BEEHR, 2018). Aspectos pessoais como gênero, idade e formação também podem afetar a percepção do profissional sobre diversos aspectos organizacionais, no que tange a políticas e ações da organização. Estas podem ser percebidas em direções e níveis diferentes, de forma que a percepção dos trabalhadores sobre sua qualidade de vida na empresa pode variar.

Assim como elementos referentes aos vínculos organizacionais podem influenciar a qualidade de vida no trabalho (MILHOME; ROWE, 2016; HUANG; LAWLER; LEI, 2007), a percepção desta última pode influenciar a natureza e o nível dos vínculos com a organização. A qualidade de vida no trabalho é compreendida como um meio de humanizar o trabalho, a partir da preocupação com a satisfação, o bem-estar e a saúde do trabalhador. Está relacionada, segundo Walton (1973), com a remuneração, as condições e estrutura de trabalho, as relações interpessoais, o reconhecimento e possibilidades de crescimento, a influência da vida profissional na pessoal e a imagem que o trabalhador tem da organização.

A formação dessa imagem diz respeito, dentre outras coisas, ao orgulho de ser parte da organização, uma característica marcante na compreensão do comprometimento organizacional. No modelo unidimensional, que vem sendo defendido nos âmbitos nacional e internacional (BASTOS; AGUIAR, 2015; SOLINGER; VAN OLFFEN; ROE, 2008) como o mais apropriado para entender efetivamente o comprometimento organizacional, este consiste em um elo afetivo entre trabalhador e organização, caracterizado: a) pelo compartilhamento de 
valores e objetivos; b) pela aceitação de normas; e c) por um comportamento do trabalhador em prol da organização.

Milhome e Rowe (2016) confirmaram influência, positiva e variando entre moderada e forte, do comprometimento organizacional em algumas dimensões da qualidade de vida no trabalho, bem como verificaram a influência de uma dimensão do entrincheiramento organizacional (ajustes burocráticos impessoais) em uma dimensão da qualidade de vida. Outros estudos evidenciaram correlação (SCHIRRMEISTER; LIMONGI-FRANÇA, 2012), predição (CELMECE et al., 2017; ZANARDI, 2016) e influência (HUANG; LAWLER; LEI, 2007; LIRIO; SEVERO; GUIMARÃES, 2018) da qualidade de vida no trabalho sobre o comprometimento organizacional, bem como deste sobre aquela. Com base nas relações encontradas na literatura, são apresentadas as seguintes hipóteses a serem testadas.

H1: trabalhadores com maior nível de qualidade de vida no trabalho percebem maior nível de comprometimento organizacional.

H2: trabalhadores com maior nível de comprometimento organizacional percebem maior nível de qualidade de vida no trabalho.

Por outro lado, elementos da qualidade de vida no trabalho como remuneração, condições e estrutura de trabalho, relações interpessoais e reconhecimento podem comprometer ou entrincheirar o trabalhador. Tais variáveis estão relacionadas: a) à necessidade de segurança financeira - ajustes burocráticos impessoais; ou b) ao medo de perder quer contatos, quer a posição alcançada na organização - ajustamento à posição social. São variáveis que caracterizam o entrincheiramento.

A partir das relações observadas na literatura, apresentadas nesta seção, serão testadas as seguintes hipóteses.

H3: trabalhadores com maior nível de qualidade de vida no trabalho percebem menor nível de entrincheiramento organizacional.

H4: trabalhadores com maior nível de entrincheiramento organizacional percebem menor nível de qualidade de vida no trabalho. 
Quadro 1 - Autores que embasaram as hipóteses da pesquisa

\begin{tabular}{|c|c|}
\hline Hipóteses & Autores \\
\hline $\begin{array}{l}\text { H1: trabalhadores com maior nível de } \\
\text { qualidade de vida no trabalho percebem } \\
\text { maior nível de comprometimento } \\
\text { organizacional. }\end{array}$ & \multirow{2}{*}{$\begin{aligned} & \text { - } \text { ALZUBI (2018); } \\
& \text { - } \text { CELMECE et al., (2017); } \\
& \text { - } \text { GUTIERREZ; CANDELA; CARVER } \\
&(2012) ; \\
& \text { - } \text { KARAKUŞ (2018); } \\
& \text { - } \text { HUANG; LAWLER; LEI (2007); } \\
& \text { - SCHIRRMEISTER; LIMONGI-FRANÇA } \\
& \text { (2012); } \\
& \text { - ZANARDI (2016); } \\
& \text { - LIRIO; SEVERO; GUIMARÃES (2018). }\end{aligned}$} \\
\hline $\begin{array}{l}\mathrm{H} 2 \text { : trabalhadores com maior nível de } \\
\text { comprometimento } \\
\text { percebem maior nível de qualidade de } \\
\text { vida no trabalho. }\end{array}$ & \\
\hline $\begin{array}{l}\text { H3: trabalhadores com maior nível de } \\
\text { qualidade de vida no trabalho percebem } \\
\text { menor nível de entrincheiramento } \\
\text { organizacional. }\end{array}$ & \multirow{2}{*}{$\begin{array}{l}\text { - } \text { ALZUBI (2018); } \\
\text { - } \text { BALSAN et al., (2015); } \\
\text { - } \text { KIM; BEEHR (2018); MEIRELES (2015); } \\
\text { - MEYER; HERSCOVITCH (2001); } \\
\text { - MEYER; STANLEY; PARFYONOVA } \\
\text { (2012). }\end{array}$} \\
\hline $\begin{array}{l}\text { H4: trabalhadores com maior nível de } \\
\text { entrincheiramento } \\
\text { percebem menor nível de qualidade de } \\
\text { vida no trabalho. }\end{array}$ & \\
\hline
\end{tabular}

Fonte: elaboração própria.

As referidas hipóteses serão testadas empiricamente, conforme apresentado nas próximas seções.

\section{METODOLOGIA}

Este estudo é classificado como quantitativo e explicativo, na medida em que propõe identificar fatores para a ocorrência de certos fenômenos, aprofundando o conhecimento da realidade, a partir da explicação do porquê das coisas (SOUZA; SANTOS; DIAS, 2013). O survey, a estratégia metodológica selecionada, baseou-se na aplicação de questionários tanto em versão on-line, distribuída por e-mail e por rede social, com a utilização da plataforma SurveyMonkey, quanto em versão impressa, entregue diretamente a respondentes potenciais.

O questionário empregou uma escala do tipo Likert - intervalar, variando entre a discordância (menor valor) e a concordância (maior valor), podendo ou não conter um ponto neutro. Apesar de Hair Jr. et al. (2009) recomendarem a retirada do ponto neutro para aumentar a fidedignidade da escala, esta pesquisa utilizou as escalas originais validadas para o contexto brasileiro.

Além dos dados sociodemográficos, o questionário foi composto por três escalas: 
a) Avaliação da Qualidade de Vida no Trabalho, desenvolvida por Walton (1973) e validada para trabalhadores brasileiros por Timossi et al. (2009), composta originalmente de 35 itens e contendo uma escala Likert de 5 pontos $-\alpha=0,94$;

b) Medida de Comprometimento Organizacional (MCO), validada para trabalhadores brasileiros por Bastos e Aguiar (2015), composta de 12 itens e de escala Likert de 6 pontos $-\alpha=0,94$; e

c) Medida de Entrincheiramento Organizacional (MEO), validada para trabalhadores brasileiros por Rodrigues e Bastos (2015), composta de 18 itens e de escala Likert de 6 pontos $-\alpha=0,89$.

Uma vez inserido o questionário na plataforma SurveyMonkey, realizou-se pré-teste com cinco trabalhadores de diversas áreas de atuação, escolhidos por critério de acessibilidade. Não sendo sinalizadas inconsistências e/ou observações relevantes que exigissem alterações significativas no instrumento, seguiu-se com a coleta dos dados, entre os meses de setembro e dezembro de 2015.

Ao todo, foram respondidos 284 questionários. Analisaram-se missing values e outliers, a fim de chegar à amostra final, composta por 187 respostas. A partir dela, realizou-se regressão múltipla para estudar a relação entre os construtos.

A regressão múltipla consiste em uma técnica estatística utilizada para analisar a relação entre duas ou mais variáveis independentes e uma dependente (HAIR JR. et al., 2009; MALHOTRA, 2012). Para que se pudesse considerá-la a solução adequada, foram realizadas algumas verificações: coeficiente de verificação da variância explicada $\left(\mathrm{R}^{2}\right)$, significância dos parâmetros com teste t e análise dos resíduos.

O coeficiente de verificação da variância explicada $\left(\mathrm{R}^{2}\right)$ indica o poder explicativo da regressão. Varia entre 0 e 1, sendo maior o poder explicativo quanto mais próximo de 1 . Além disso, através do teste $\mathrm{t}$, verificou-se o coeficiente angular para cada $\beta$ da regressão. Por fim, analisaram-se os resíduos a partir da homocedasticidade, linearidade, normalidade e independência.

Dentre os 187 questionários válidos para análise, 7 não tiveram preenchidas as informações dos dados sociodemográficos, de forma que não puderam compor a caracterização da amostra analisada. Os trabalhadores são, em sua maioria, do sexo feminino (56\%), com idade média de 31 anos (DP: 8,78), solteiros (56\%), graduados (39\%) e com especialização (37\%), atuando no setor privado (71\%), com tempo médio de serviço de 5 anos (DP: 6,82). 


\section{RESULTADOS}

Partindo do objetivo central deste estudo, apresentam-se os resultados provenientes da análise dos dados obtidos através da técnica de regressão múltipla. A fim de entender as relações que se estabelecem entre qualidade de vida no trabalho, $\mathrm{CO}$ e entrincheiramento organizacional, analisou-se a influência de cada uma das 8 dimensões da qualidade de vida no trabalho no CO (unidimensional) e em cada uma das 3 dimensões do entrincheiramento organizacional, bem como se examinou a influência do CO (unidimensional) e de cada uma das 3 dimensões do entrincheiramento organizacional em cada uma das 8 dimensões da qualidade de vida no trabalho. Para tanto, foram realizadas 12 análises de regressão múltipla.

São dimensões da qualidade de vida no trabalho: remuneração justa e adequada (RJA), condições de trabalho (CDT), uso e desenvolvimento das capacidades de trabalho (CPT), oportunidade de crescimento e segurança (OPT), integração social na organização (INT), constitucionalismo (CON), o trabalho e o espaço total na vida (ETV) e relevância social do trabalho na vida (RST). São dimensões do entrincheiramento organizacional: ajustamento à posição social (APS), limitação de alternativas (LA) e ajustes burocráticos impessoais (ABI). O comprometimento organizacional é compreendido, neste estudo, como um vínculo unidimensional, representado pela sigla $\mathrm{CO}$ ao longo dos resultados e discussões.

Os resultados das próximas sessões apresentam, ao todo, seis modelos estatisticamente válidos, os quais encontram suporte teórico na literatura anteriormente abordada.

\subsection{Influência da qualidade de vida no trabalho nos vínculos com a organização}

Foram testados quatro modelos relacionando as dimensões da qualidade de vida no trabalho (variáveis independentes) a cada uma das quatro dimensões dos vínculos (variável dependente). No entanto, conforme indicam os dados: a) somente uma dimensão da qualidade de vida no trabalho explica, com significância e validade estatística, uma dimensão do entrincheiramento organizacional; e b) três dimensões da qualidade de vida no trabalho explicam, com significância e validade estatística, o CO.

A dimensão LA - do entrincheiramento organizacional - apresentou variação conjunta somente com a RJA - dimensão da qualidade de vida no trabalho. Trata-se de uma relação fraca $(\mathrm{R}=0,28)$. Conjuntamente, as variáveis da qualidade de vida no trabalho explicam em $4 \%$ a 
percepção de LA. No entanto, somente a variável RJA apresenta uma relação estatisticamente significativa, conforme se pode verificar na Tabela 1.

Tabela 1 - Parâmetros e testes para a regressão 1 - influência da qualidade de vida no trabalho sobre o entrincheiramento organizacional

\begin{tabular}{|c|c|c|c|c|c|c|c|c|c|}
\hline \multirow[t]{2}{*}{ Variáveis } & \multicolumn{2}{|c|}{ Estimadores } & \multicolumn{2}{|c|}{ Teste t do estimador } & \multicolumn{5}{|c|}{ Grau de ajustamento } \\
\hline & $\begin{array}{c}\text { Estimador } \\
\beta\end{array}$ & $\begin{array}{c}\text { Erro } \\
\text { padrão }\end{array}$ & t-valor & Sig. & F & Sig. & $\mathrm{R}$ & $\overline{\mathrm{R}^{2}}$ & $\begin{array}{c}\mathrm{R}^{2} \\
\text { ajustado }\end{array}$ \\
\hline Intercepto & 2,990 & 0,450 & 6,645 & $3,57 \mathrm{e}-10$ & \multirow{9}{*}{1,95} & \multirow{9}{*}{0,05} & \multirow{9}{*}{0,28} & \multirow{9}{*}{0,08} & \multirow{9}{*}{0,04} \\
\hline RJA & 0,264 & 0,109 & 2,412 & 0,0169 & & & & & \\
\hline CDT & $-0,044$ & 0,167 & $-0,266$ & 0,7908 & & & & & \\
\hline CPT & $-0,147$ & 0,160 & $-0,919$ & 0,3591 & & & & & \\
\hline OPT & $-0,127$ & 0,139 & $-0,915$ & 0,3616 & & & & & \\
\hline INT & $-0,082$ & 0,161 & $-0,508$ & 0,6123 & & & & & \\
\hline $\mathrm{CON}$ & $-0,212$ & 0,166 & $-1,274$ & 0,2043 & & & & & \\
\hline ETV & 0,025 & 0,124 & 0,206 & 0,8374 & & & & & \\
\hline RST & 0,137 & 0,154 & 0,887 & 0,3762 & & & & & \\
\hline
\end{tabular}

Fonte: elaboração própria.

Sig.: 0,05

A RJA diz respeito à percepção de que a remuneração é justa, se comparada com os colegas de trabalho e o mercado, e adequada para atender às necessidades do trabalhador. A LA, por sua vez, refere-se à percepção de que o mercado nem oferece alternativas condizentes com a atuação específica do trabalhador nem capazes de evitar perdas, o que gera nele a sensação de estar preso à organização. Uma possível motivação para tal sentimento é a remuneração se, por exemplo, a saída do trabalhador pode acarretar perdas em termos de remuneração e benefícios. No entanto, os resultados não confirmam a hipótese H3, pois esta verifica uma relação negativa entre qualidade de vida no trabalho e entrincheiramento organizacional e a única relação estatisticamente válida apresentada foi positiva.

Por sua vez, a forte relação $(0,78)$ entre a qualidade de vida no trabalho e o CO também era esperada com base na literatura. Porém, diversas dimensões da qualidade de vida no trabalho não apresentaram relação com o $\mathrm{CO}$, assim confirmando parcialmente a hipótese H1. A variação no nível de qualidade de vida no trabalho explica em 59\% a variação no nível de CO. As variáveis com relação estatisticamente significativa, conforme é possível verificar na Tabela 2, são: RJA (t-valor: 2,530; sig.: 0,01); CON (t-valor: 5,235; sig.: 4,62 e-07); RST (t-valor: 2,676; sig.: 0,01). 
Tabela 2 - Parâmetros e testes para a regressão 2 - influência da qualidade de vida no trabalho sobre o $\mathrm{CO}$

\begin{tabular}{|c|c|c|c|c|c|c|c|c|c|}
\hline \multirow[t]{2}{*}{ Variáveis } & \multicolumn{2}{|c|}{ Estimadores } & \multicolumn{2}{|c|}{ Teste t do estimador } & \multicolumn{5}{|c|}{ Grau de ajustamento } \\
\hline & Estimador $\beta$ & Erro padrão & t-valor & Sig. & $\mathrm{F}$ & Sig. & $\mathrm{R}$ & $\mathrm{R}^{2}$ & $\mathrm{R}^{2}$ ajustado \\
\hline Intercepto & $-0,190$ & 0,309 & $-0,616$ & 0,54 & \multirow{9}{*}{34,98} & \multirow{9}{*}{$2,2 \mathrm{e}-16$} & \multirow{9}{*}{0,78} & \multirow{9}{*}{0,61} & \multirow{9}{*}{0,59} \\
\hline RJA & 0,190 & 0,075 & 2,530 & 0,01 & & & & & \\
\hline CDT & $-0,038$ & 0,115 & $-0,329$ & 0,74 & & & & & \\
\hline CPT & 0,115 & 0,110 & 1,046 & 0,30 & & & & & \\
\hline OPT & 0,059 & 0,096 & 0,615 & 0,54 & & & & & \\
\hline INT & 0,027 & 0,110 & 0,245 & 0,81 & & & & & \\
\hline $\mathrm{CON}$ & 0,597 & 0,114 & 5,235 & $4,62 \mathrm{e}-07$ & & & & & \\
\hline ETV & $-0,027$ & 0,085 & $-0,327$ & 0,74 & & & & & \\
\hline RST & 0,283 & 0,106 & 2,676 & 0,01 & & & & & \\
\hline
\end{tabular}

Fonte: elaboração própria.

Sig.: 0,05

O CO diz respeito ao orgulho de pertencer à organização e ao compartilhamento de normas e valores. A literatura aponta a relação entre RJA e comprometimento, enquanto a satisfação com remuneração é um forte preditor do $\mathrm{CO}$. Ao compreender o $\mathrm{CO}$ a partir do orgulho de pertencer à organização e do compartilhamento de normas e valores, inferia-se que o trabalhador compartilharia normas condizentes com a legalidade.

No entanto, não se esperava uma relação tão forte. O estimador $\beta$ é o maior deste modelo, sugerindo, em termos estatísticos, que o aumento de 1 ponto na percepção de CON aumenta em 0,597 a percepção de CO, com confiança superior a 99\%. Acredita-se que o cumprimento da legislação trabalhista seja basilar na relação entre organização e trabalhador. No entanto, este resultado indica uma relação de baixa confiança entre o trabalhador brasileiro e a organização. Como ele aponta uma grande relação entre o cumprimento dos seus direitos e o seu nível de comprometimento, isso sugere que a relação entre organização e trabalhador precisa ser revista.

A RST, dentre outras coisas, está ligada ao orgulho de integrar a organização e à imagem que o trabalhador e a sociedade têm dela. Sendo o orgulho de pertencer um elemento preponderante no conceito do $\mathrm{CO}$, esperava-se que esta variável da qualidade de vida no trabalho se relacionasse fortemente e com boa significância, conforme os resultados demonstraram. 


\subsection{Influência dos vínculos com a organização na qualidade de vida no trabalho}

Para esta análise, testaram-se oito modelos, por regressão múltipla, relacionando os vínculos (variáveis independentes) a cada uma das variáveis que compõem a qualidade de vida no trabalho.

O terceiro modelo testado na pesquisa relacionou as três dimensões do entrincheiramento organizacional (APS, LA, ABI) e o CO à dimensão RJA, da qualidade de vida no trabalho, como variável dependente. Conjuntamente, os vínculos explicam $41 \%$ da variação da percepção de RJA. No entanto, somente as dimensões LA, ABI e CO apresentam significância estatística nesta variação (Tabela 3).

Tabela 3 - Parâmetros e testes da regressão 3 - influência dos vínculos sobre a RJA

\begin{tabular}{l|l|l|l|l|l|l|l|l|l}
\hline \multirow{2}{*}{ Variáveis } & \multicolumn{3}{|c|}{ Estimadores } & \multicolumn{2}{c|}{ Teste t do estimador } & \multicolumn{5}{c}{ Grau de ajustamento } \\
\cline { 2 - 8 } & Estimador $\beta$ & Erro padrão & t-valor & Sig. & $\mathrm{F}$ & Sig. & $\mathrm{R}$ & $\mathrm{R}^{2}$ & $\mathrm{R}^{2}$ ajustado \\
\hline Intercepto & 0,671 & 0,243 & 2,766 & 0,006 & & & & & \\
\hline APS & 0,088 & 0,062 & 1,403 & 0,162 & & & & & \\
\hline LA & $-0,118$ & 0,059 & $-1,997$ & 0,047 & \multirow{2}{*}{2,91} & 0,00 & 0,65 & 0,42 & 0,41 \\
\hline ABI & 0,255 & 0,053 & 4,819 & 0,000 & & & & & \\
\hline CO & 0,403 & 0,504 & 8,012 & 0,000 & & & & & \\
\hline
\end{tabular}

Fonte: elaboração própria.

Sig.: 0,05

Conforme se percebe da análise dos estimadores $\beta$, a variável CO é a que mais explica a percepção de RJA, na relação analisada. Essa era uma relação já esperada, pois a literatura mostra o CO com forte influência na satisfação com remuneração. Essa é uma relação direta, ou seja, o aumento do CO melhora a percepção de justiça e adequação da remuneração.

A variável ABI - Arranjos Burocráticos Impessoais - apresenta poder explicativo menor, porém significativo. Para esta variável, como se trata de perdas associadas à remuneração, esperava-se o resultado apresentado. Ou seja, maior sendo a percepção de perdas ligadas à ABI, igualmente maior será a percepção de RJA.

Por sua vez, observa-se que a percepção de se sentir preso à organização, resultante da variável LA, está inversamente relacionada à variável dependente. Dessa forma, o aumento da LA se relaciona inversamente com uma menor percepção de RJA.

A quarta regressão analisou as variáveis independentes supracitadas explicando a variável dependente CDT, dimensão da qualidade de vida no trabalho. Este modelo explica $32 \%$ da variação da percepção acerca das condições adequadas de trabalho. No entanto, 
somente a variável CO apresenta significância estatística para explicar o modelo. A Tabela 4 apresenta os parâmetros e testes para esta regressão.

Tabela 4 - Parâmetros e testes da regressão 4 - influência dos vínculos sobre a CDT

\begin{tabular}{l|l|l|l|l|l|l|l|l}
\hline \multirow{2}{*}{ Variáveis } & \multicolumn{3}{|c|}{ Estimadores } & \multicolumn{2}{c|}{ Teste t do estimador } & \multicolumn{4}{c}{ Grau de ajustamento } \\
\cline { 2 - 5 } & Estimador $\beta$ & Erro padrão & t-valor & Sig. & F & Sig. & $\mathrm{R}^{2}$ & $\mathrm{R}^{2}$ ajustado \\
\hline Intercepto & 1,921 & 0,220 & 8,714 & 0,000 & & & & \\
\hline APS & 0,084 & 0,057 & 1,470 & 0,143 & & & & \\
\hline LA & $-0,104$ & 0,054 & $-1,932$ & 0,055 & 23,19 & 0,000 & 0,34 & 0,32 \\
\hline ABI & 0,033 & 0,048 & 0,618 & 0,497 & & & & \\
\hline CO & 0,356 & 0,046 & 7,779 & 0,000 & & & & \\
\hline
\end{tabular}

Fonte: elaboração própria.

Sig.: 0,05

CDT, neste contexto, é compreendido como jornada e carga de trabalho, segurança e salubridade no trabalho, cansaço proveniente do trabalho. Não se identificaram na literatura indicativos de que elementos das CDT poderiam ser preditos ou influenciados por elementos pertinentes ao CO. No entanto, esse resultado sugere que um maior nível de CO favorece uma melhor percepção das CDT.

A análise da quinta regressão considerou, como variável dependente, equilíbrio entre trabalho e vida (ETV), dimensão da qualidade de vida no trabalho. Os vínculos analisados explicam $22 \%$ da variação na percepção acerca do equilíbrio entre vida pessoal e trabalho. A Tabela 5 apresenta os parâmetros e testes para esta regressão.

Tabela 5 - Parâmetros e testes da regressão 5 - influência dos vínculos sobre a dimensão ETV

\begin{tabular}{l|l|l|l|c|c|c|c|c}
\hline \multirow{2}{*}{ Variáveis } & \multicolumn{2}{|c|}{ Estimadores } & \multicolumn{2}{c|}{ Teste t do estimador } & \multicolumn{2}{c|}{ Estatística $\mathrm{F}$} & \multicolumn{2}{c}{ Grau de ajustamento } \\
\cline { 2 - 7 } & Estimador $\beta$ & Erro padrão & $\mathrm{t}$-valor & $\mathrm{Sig}$ & $\mathrm{F}$ & $\mathrm{Sig}$ & $\mathrm{R}^{2}$ & $\mathrm{R}^{2}$ ajustado \\
\hline Intercepto & 1,956 & 0,270 & 7,254 & 0,000 & 13,86 & 0,00 & 0,23 & 0,22 \\
\hline APS & 0,089 & 0,070 & 1,271 & 0,205 & & & & \\
\hline LA & $-0,128$ & 0,070 & $-1,936$ & 0,054 & & & & \\
\hline ABI & 0,068 & 0,059 & 1,160 & 0,248 & & & & \\
\hline CO & 0,322 & 0,056 & 5,742 & 0,000 & & & & \\
\hline
\end{tabular}

Fonte: elaboração própria.

Sig.: 0,05

Mais uma vez, somente a variável independente $\mathrm{CO}$ explica a variável dependente com significância estatística. O equilíbrio entre a vida pessoal e o trabalho está ligado ao bem-estar do trabalhador, que inclui entre seus componentes o $\mathrm{CO}$. No sentido contrário, ou seja, o $\mathrm{CO}$ predizendo o bem-estar do trabalhador e equilíbrio entre vida pessoal e trabalho, autores como Mathieu e Zajac (1990), Cooper-Hakim e Viswevaran (2005) e Traldi e Demo (2012) permitem inferir a relação confirmada por estes resultados. 
A sexta regressão objetivou compreender o grau de explicação das variáveis independentes na variável dependente RST, dimensão da qualidade de vida no trabalho. A variação na percepção da variável dependente é explicada em $42 \%$ por este modelo (Tabela 6).

Tabela 6 - Parâmetros e testes da regressão 6 - influência dos vínculos sobre a dimensão ETV

\begin{tabular}{l|l|l|l|l|l|l|l|l}
\hline \multirow{2}{*}{ Variáveis } & \multicolumn{3}{|c|}{ Estimadores } & \multicolumn{2}{c|}{ Teste t do estimador } & \multicolumn{2}{c|}{ Estatística $\mathrm{F}$} & \multicolumn{2}{c}{ Grau de ajustamento } \\
\cline { 2 - 6 } & Estimador $\beta$ & Erro padrão & $\mathrm{t}$-valor & Sig & $\mathrm{F}$ & $\mathrm{Sig}$ & $\mathrm{R}^{2}$ & $\mathrm{R}^{2}$ ajustado \\
\hline Intercepto & 1,837 & 0,198 & 9,259 & 0,00 & 35,09 & 0,00 & 0,44 & 0,42 \\
\hline APS & 0,137 & 0,051 & 2,664 & 0,01 & & & & \\
\hline LA & $-0,075$ & 0,049 & $-1,542$ & 0,12 & & & & \\
\hline ABI & 0,004 & 0,043 & 0,096 & 0,92 & & & & \\
\hline CO & 0,384 & 0,041 & 9,336 & 0,00 & & & & \\
\hline
\end{tabular}

Fonte: elaboração própria.

Sig.: 0,05

Tal como indicam os resultados, a RST é influenciada pelas variáveis CO e APS. A variável CO explica da melhor maneira a variável dependente $(b=0,38, p<0,05)$. Como a RST está ligada tanto ao orgulho de pertencer à organização, quanto à imagem que o trabalhador tem dela, esperava-se uma forte relação entre as variáveis analisadas, em ambos os sentidos: CO influenciando RST e RST influenciando CO, tal como confirmado pelos resultados.

Em relação às dimensões do entrincheiramento organizacional, somente o APS $(b=0,14$, $\mathrm{p}<0,05)$ explica significativamente a influência do entrincheiramento organizacional na percepção de RST. Diante dos poucos estudos que relacionam entrincheiramento organizacional com os diversos construtos, não existiam indicativos dessa relação na literatura, assim como as definições destas não apresentam possíveis relações claras.

A variável APS é definida por Rodrigues e Bastos (2015) como “investimento do indivíduo e da organização em condições necessárias para o bom desempenho de determinada atividade e adaptação do trabalhador a [sic] posição em que se encontra”. Está ligado, ainda, às expectativas dos diversos atores sociais e às relações sociais estabelecidas na organização. É possível inferir desse resultado que, quanto mais o trabalhador se percebe ligado socialmente à organização, maior o seu orgulho de a ela pertencer e melhor a imagem que constrói acerca dela. No entanto, não há relação significativa entre as variáveis LA e RST.

A análise dos resíduos das regressões foi determinante para compreender a adequação dos modelos investigados para explicar o fenômeno estudado. Foram analisados, para tanto, normalidade, independência, homocedasticidade e linearidade dos resíduos. As quatro regressões pormenorizadas possuem índices indicativos de adequação dos modelos. Em contrapartida, outros quatro modelos, cujas variáveis dependentes foram Uso das Capacidades no Trabalho, Oportunidades no Trabalho, Integração no Trabalho e CON, apresentaram índices 
que apontam para modelos não explicativos da relação estudada. Os resultados apresentados estão sintetizados na Figura 1.

Figura 1 - Relações encontradas no estudo

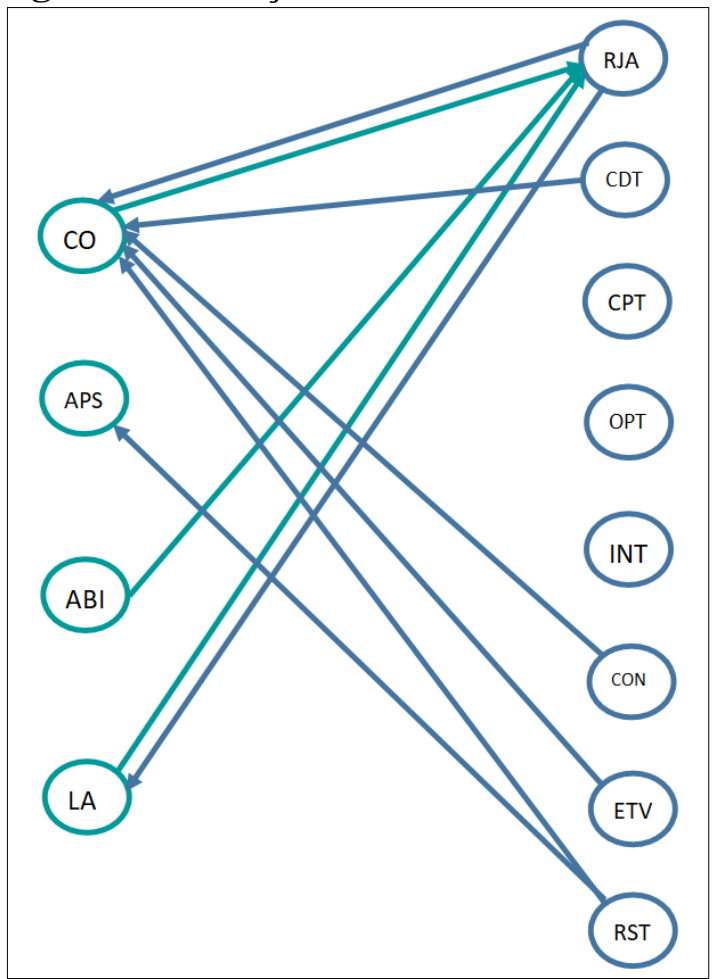

Fonte: elaboração própria.

O CO é o que melhor explica as variáveis dependentes analisadas em todos os modelos propostos, conforme já indicado pela literatura e demonstrado no estudo empírico. No entanto, não se verificaram relações com algumas dimensões, confirmando-se parcialmente a hipótese $\mathrm{H} 2$.

Características pessoais e organizacionais têm um impacto direto menor, assim como experiências de trabalho têm um impacto maior no CO (BASTOS et al., 2013). Em alguma medida, esses podem afetar o bem-estar, a satisfação e a lealdade do trabalhador (GUTIERREZ; CANDELA; CARVER, 2012). O bem-estar e a satisfação do trabalhador estão diretamente relacionados com a qualidade de vida no trabalho. Por sua vez, a lealdade mostra-se importante na percepção da imagem da organização, que faz parte do construto "qualidade de vida no trabalho", especificamente na dimensão RST.

Os modelos de regressão apresentados corroboram a literatura, apontando que a satisfação com aspectos do trabalho como remuneração, ambiente e CDT se relacionam com o comprometimento do trabalhador. Além disso, o comprometimento do trabalhador contribui 
para ter orgulho de integrar a organização e para possuir uma imagem positiva desta, percebendo como mais equilibrada a relação entre trabalho e vida pessoal.

No que tange ao entrincheiramento organizacional, dimensões do construto explicam algumas variáveis dependentes em proporção menor que o CO. Os resultados apresentam as variáveis independentes ABI e LA influenciando significativamente a percepção de RJA. Essa relação foi apontada na literatura, a exemplo de Gutierrez, Candela e Carver (2012), e confirmada no estudo empírico. A variável ABI está ligada às perdas financeiras, dessa forma, interferindo na percepção da justiça no que se refere a benefícios e a remuneração.

Por sua vez, a variável LA diz respeito à percepção limitada de alternativas que permitam ao trabalhador sair da trincheira e apresenta relação inversa. Esperava-se a significância desta em todas as regressões, pois a sensação de prisão é determinante para a existência do entrincheiramento organizacional, influenciando negativamente a percepção da qualidade de vida no trabalho, a partir das suas dimensões constitutivas. No entanto, ao contrário do que apontava a hipótese $\mathrm{H} 4$, mostrou-se significativa somente na primeira regressão.

Existem poucas relações evidenciadas entre as variáveis dependentes e aquelas relativas ao entrincheiramento organizacional. Por isso, recomendam-se novos estudos para aprofundar essas relações. Até que ponto é interessante incentivar, no trabalhador, a percepção de custos de saída? Apesar de a influência destes na percepção de qualidade de vida no trabalho ser positiva, somente o custo relacionado à perda de estabilidade financeira influencia a qualidade de vida no trabalho. Mais precisamente, a percepção de RJA possui peso substancial que justifica o incentivo a perceber tais custos.

Demonstra-se, então, a partir desses modelos e das reflexões propostas, a importância de conhecer as diversas relações e influências que permeiam o vínculo do trabalhador com a organização e podem, por consequência, interferir na efetividade e no sucesso organizacional.

\section{CONSIDERAÇÕES FINAIS}

Compreender as diversas relações que permeiam o campo da gestão de pessoas contribui para buscar um gerenciamento mais atuante em termos humanos, com resultados melhores. Dentre tais relações, esta pesquisa visou estudar aquela entre, de um lado, os vínculos comprometimento e entrincheiramento organizacional e, do outro, a qualidade de vida no trabalho, enquanto antecedentes e consequentes. 
Para compreender essa relação, utilizou-se regressão múltipla, testando 12 modelos, sendo seis deles estatisticamente adequados para analisar as relações investigadas. Os resultados evidenciaram relações de todas as dimensões dos vínculos com quase todas as dimensões da qualidade de vida no trabalho. Somente as dimensões CPT, OPT e INT não se relacionaram significativamente com os vínculos.

O entrincheiramento organizacional, como percepção de estar preso à organização por conta das possíveis perdas ligadas à saída, é compreendido a partir de três dimensões: APS, ABI e LA (RODRIGUES; BASTOS, 2015), as quais explicam variações na forma de perceber duas dimensões da qualidade de vida no trabalho: RJA e RST.

Os resultados confirmaram relações já esperadas com base na literatura, tais como a ligação direta de $\mathrm{ABI}$ à percepção de RJA e o fato de esta última explicar e ser explicada pela LA. Também emergiu uma relação que ainda não havia sido verificada, mostrando-se interessante conduzir estudo futuro para esclarecer o fenômeno.

No entanto, esperavam-se relações inversas, no caso da qualidade de vida no trabalho e do entrincheiramento organizacional. Como, dentre as quatro relações estatisticamente significativas apresentadas, somente uma se comportou daquela maneira, as hipóteses H3 e H4 não foram confirmadas.

Por sua vez, o CO relaciona-se direta e positivamente com as dimensões ETV e CDT, bem como prediz e é predito pelas dimensões RST e RJA, confirmando algumas relações inferidas a partir da literatura. Porém, uma relação que, apesar de ser indicada pela literatura, não se esperava tão forte é a influência de $\mathrm{CON}$ sobre a percepção do $\mathrm{CO}$, o que sugere a utilidade de repensar a relação trabalhador-empresa.

Esperava-se que todas as relações evidenciadas entre qualidade de vida no trabalho e $\mathrm{CO}$ fossem diretas. No entanto, houve mais relações indicando uma influência do CO na percepção da qualidade de vida no trabalho do que no sentido contrário. Esta pesquisa, então, confirma apenas parcialmente as hipóteses $\mathrm{H} 1$ e $\mathrm{H} 2$.

Seja como for, a satisfação também está ligada a aspectos como personalidade e valores do profissional, variáveis estas não abarcadas pelo presente estudo, sinalizando portanto a relevância de investigações futuras. Em contrapartida, esta contribuiu com a indicação ou confirmação de relações não apresentadas em estudos anteriores e/ou inferidos na literatura, 
permitindo compreender melhor as relações existentes entre os construtos estudados. Todavia, diante de algumas limitações, como o tamanho da amostra, sugerem-se novas pesquisas com amostra maior, visando a análises mais consistentes.

Os achados aqui expostos contribuem para a melhor compreensão dos construtos, como já era esperado. Mas, além disso, a relação entre as dimensões permitiu refletir acerca dos elementos da organização que podem ser importantes para desenvolver e manter os vínculos do indivíduo com a organização. Analisar os elementos organizacionais que representam cada uma das dimensões da qualidade de vida no trabalho permite perceber, a partir dos resultados, o quanto cada um desses elementos pode impactar o desenvolvimento e manutenção dos vínculos com a organização.

Por outro lado, uma análise conceitual das dimensões dos vínculos trouxe o que cada uma delas representa em termos de ligação do indivíduo com a organização. Dessa forma, ajuda a perceber como esses elementos, que conjuntamente representam vínculos com a organização de diferentes naturezas, podem ser significativos na percepção, construída pelo trabalhador, da sua qualidade de vida no trabalho. Diversas pesquisas (CARVALHO-FREITAS; MARQUES; ALMEIDA, 2009; SCHIRRMEISTER; LIMONGI-FRANÇA, 2012; ZANARDI, 2016) buscam compreender o impacto da organização sobre o trabalhador. O presente estudo, por sua vez, lançou luz sobre o modo como uma organização contribui para o vínculo do trabalhador com ela, colocando-a como corresponsável pelos efeitos tanto positivos - provenientes do CO -, quanto dos negativos, advindos do entrincheiramento organizacional. Contudo, o sentido oposto indica que, mesmo se a organização apresentar estímulos equânimes, a efetividade deles perpassará a percepção do trabalhador acerca deles e o que cada estímulo representa na perspectiva desse trabalhador.

Fica reforçada a importância de futuros estudos se basearem em uma perspectiva em que o trabalhador participe de modo substancial do processo de formação e manutenção do vínculo, tanto quanto ou até mais que a organização. Recomenda-se, ainda, que os vínculos, conjuntamente, sejam relacionados com outros construtos, tanto enquanto antecedentes como consequentes ou mesmo mediadores de uma relação. Especialmente o conceito de entrincheiramento organizacional, novo, ainda carece de pesquisas para compreender a sua influência e a sua relação com outros construtos, contando ou não com a influência do CO. Isso poderá auxiliar no desenvolvimento e na consistência do construto, bem como na gestão de pessoas, podendo contribuir para melhores desempenhos organizacionais. 


\section{REFERÊNCIAS}

ALZUBI, Y. Z. W. Turnover intentions in Jordanian Universities: The role of leadership behaviour, organizational commitment and organizational culture. International Journal of Advanced and Applied Sciences, v. 5, n. 1, p. 177-192, 2018.

BALSAN, L. A. G.; BASTOS, A. V. B.; FOSSÁ, M. I. T.; LIMA, M. P.; LOPES, L. F. D.; COSTA, V. M. F. Comprometimento e Entrincheiramento Organizacional: Explorando as Relações entre os Construtos. Revista de Administração da UFSM, v. 8, n. 2, p. 235-248, 2015.

BASTOS, A. V. B.; AGUIAR, C. V. N. Comprometimento Organizacional. In: PUENTEPALACIOS, K.; PEIXOTO, A. L. A. (Org.). Ferramentas de diagnóstico para organizações e trabalho: Um olhar a partir da psicologia. Porto Alegre: Artmed, 2015.

; RODRIGUES, A. C. A.; MOSCON, D. C. B.; SILVA, E. E. C.; PINHO, A. P. M. Comprometimento no trabalho: fundamentos para a gestão de pessoas. In: BORGES, L. O.; MOURÃO, L. (Org.). O trabalho e as organizações: atuações a partir da psicologia. Porto Alegre: Artmed, 2013.

CARVAlHO-FREITAS, M. N.; MARQUES, A. L.; ALMEIDA, L. A. D. Pessoas com deficiência: comprometimento organizacional, condições de trabalho e qualidade de vida no trabalho. Gerais: Rev. Interinst. Psicol., Juiz de fora, v. 2, n. 2, p. 92-105, 2009.

CELMECE, N.; ISHIKLAR, A.; MACAR, M.; KAYA, T. Organizational Commitment as a Predictor of Job Life Quality. Khazar Journal of Humanities and Social Sciences, p. 92-102, 2017

COOPER-HAKIM, A.; VISWESVARAN, C. The construct of work commitment: testing an integrative framework. Psychological Bulletin, v. 131, n. 2, p. 241-259, 2005.

DEMIRTAS, O.; AKDOGAN, A. A. The effect of ethical leadership behavior on ethical climate, turnover intention, and affective commitment. Journal of Business Ethics, v. 130, n. 1, p. 59-67, 2015.

GUTIERREZ, A. P.; CANDELA, L. L.; CARVER, L. The structural relationships between organizational commitment, global job satisfaction, developmental experiences, work values, organizational support, and person-organization fit among nursing faculty. Journal of Advanced Nursing, v. 68, n. 7, p. 601-614, 2012. 
HAIR JR., J. F., ANDERSON, R. E., TATHAM, R. L.; BLACK, W. C. Análise multivariada de dados. Porto Alegre: Bookman, 2009.

HUANG, T. C.; LAWLER, J.; LEI, C. Y. The effects of Quality of Work Life on Commitment and Turnover Intention. Social Behavior and Personality: An International Journal, v. 35, n. 6, p. 735-750, 2007.

KARAKUŞ, M. The Moderating Effect of Gender on the Relationships between Age, Ethical Leadership, and Organizational Commitment. Journal of Ethnic and Cultural Studies, v. 5, n. 1, p. 74-84, 2018.

KIM, M.; BEEHR, T. A. Empowering leadership: leading people to be present through affective organizational commitment? The International Journal of Human Resource, 2018.

LIRIO, A. B.; SEVERO, E. A.; GUIMARÃES, J. C. F. A influência da Qualidade de Vida no Trabalho sobre o Comprometimento Organizacional. Revista Gestão e Planejamento, v. 19, p. 34-54, 2018.

MALHOTRA, N. Pesquisa de marketing: uma pesquisa orientada. 2. ed. Porto Alegre: Bookman, 2012.

MATHIEU, J. E.; ZAJAC, D. M. Review and meta-analysis of the antecedents, correlates, and consequences of organizational commitment. Psychological Bulletin, v. 108, n. 2, p. 171-194, 1990.

MEIRELES, A. Q. Fico porque preciso: entrincheiramento organizacional e satisfação no trabalho para empregados de uma empresa de energia brasileira. Dissertação (Mestrado em Administração) - Pontifícia Universidade Católica do Rio de Janeiro, Rio de Janeiro, Brasil, 2015.

MEYER, J. P.; HERSCOVITCH, L. Commitment in the workplace: Toward the general model. Human Resource Management Review, v. 11, p. 299-326, 2001

; STANLEY, L. J.; PARFYONOVA, N. M. Employee commitment in context: The nature and implication of commitment profiles. Journal of Vocational Behavior, v. 80, n. 1, p. $1-16,2012$

MILHOME, J. C.; ROWE, D. E. O. Comprometimento e Entrincheiramento Organizacional: possíveis correlações. XLI Encontro da ANPAD, Anais... São Paulo, Brasil, ANPAD, 2017. 
Influência dos vínculos Comprometimento e Entrincheiramento Organizacional na Qualidade de Vida no Trabalho XL Encontro da ANPAD, Anais... Costa do Sauipe, Brasil, ANPAD, 2016.

RODRIGUES, A. C. A. Do comprometimento de continuação ao entrincheiramento organizacional: o percurso de validação da escala e análise da sobreposição entre os construtos. Dissertação (Mestrado em Psicologia) - Universidade Federal da Bahia, Salvador, Brasil, 2009.

; BASTOS, A. V. B. Entrincheiramento Organizacional. In: PUENTE-PALACIOS, K.; PEIXOTO, A. L. A. (Org.). Ferramentas de diagnóstico para organizações e trabalho: um olhar a partir da psicologia. Porto Alegre: Artmed, 2015.

SCHIRRMEISTER, R.; LIMONGI-FRANÇA, A. C. A Qualidade de Vida no Trabalho: relações com o comprometimento organizacional nas equipes multicontratuais. Revista Psicologia Organizações e Trabalho, v. 12, n. 3, p. 283-298, 2012.

SOLINGER, O. N.; VAN OLFFEN, W.; ROE, R. A. Beyond the three-component model of organizational commitment. Journal of Applied Psychology, v. 93, n.1, 70-83, 2008.

SOUZA, G. S.; SANTOS, A. R.; DIAS, V. B. Metodologia da pesquisa científica: a construção do conhecimento e do pensamento científico no processo de aprendizado. São Paulo: Animal, 2013.

TIMOSSI, L. S.; PEDROSO, B.; PILATTI, L. A.; FRANCISCO, A. C. Adaptação do modelo de Walton para avaliação da qualidade de vida no trabalho. Revista da Educação Física da Universidade Estadual de Maringá, v. 20, n. 3, p. 395-405, 2009.

TRALDI, M. T. F.; DEMO, G. Comprometimento, bem-estar e satisfação dos professores de administração de uma universidade federal. Revista Eletrônica de Administração - REAd, v. 18, n. 2, p. 290-316, 2012.

WALTON, R. Quality of working life: what is it? Sloan Management Review, v. 15, n. 1, p. 11-21, 1973.

ZANARDI, E. Qualidade de Vida no Trabalho e Comprometimento Organizacional em uma empresa do setor varejista. Dissertação (Mestrado em Engenharia da Produção) Universidade Tecnológica Federal do Paraná, Curitiba, Brasil, 2016. 
ZANELLI, J. C.; BASTOS, A. V. B.; RODRIGUES, A. C. A. Campo profissional do psicólogo em organizações e no trabalho. In: ZANELLI, José Carlos; BORGES-ANDRADE, J. E.; BASTOS, A. V. B. (Org.). Psicologia, organizações e trabalho no Brasil, 2. ed. Porto Alegre: Artmed, 2014. 